\title{
Combining doxorubicin-nanobubbles and shockwaves for anaplastic thyroid cancer treatment: preclinical study in a xenograft mouse model
}

\author{
Francesca Marano1, Roberto Frairia', Letizia Rinella', Monica Argenziano², \\ Benedetta Bussolati3 , Cristina Grange', Raffaella Mastrocola4, Isabella Castellano', \\ Laura Berta5, Roberta Cavalli² and Maria Graziella Catalano' \\ 1Department of Medical Sciences, University of Turin, Turin, Italy \\ 2Department of Drug Science and Technology, University of Turin, Turin, Italy \\ 3Department of Molecular Biotechnology and Health Sciences, University of Turin, Turin, Italy \\ ${ }^{4}$ Department of Clinical and Biological Sciences, University of Turin, Turin, Italy \\ ${ }^{5}$ Med \& Sport 2000 Srl, Turin, Italy
}

Correspondence should be addressed to M G Catalano Email

mariagraziella.catalano@ unito.it

\begin{abstract}
Anaplastic thyroid cancer is one of the most lethal diseases, and a curative therapy does not exist. Doxorubicin, the only drug approved for anaplastic thyroid cancer treatment, has a very low response rate and causes numerous side effects among which cardiotoxicity is the most prominent. Thus, doxorubicin delivery to the tumor site could be an import goal aimed to improve the drug efficacy and to reduce its systemic side effects. We recently reported that, in human anaplastic thyroid cancer cell lines, combining doxorubicin-loaded nanobubbles with extracorporeal shock waves, acoustic waves used in lithotripsy and orthopedics without side effects, increased the intracellular drug content and in vitro cytotoxicity. In the present study, we tested the efficacy of this treatment on a human anaplastic thyroid cancer xenograft mouse model. After 21 days, the combined treatment determined the greatest drug accumulation in tumors with consequent reduction of tumor volume and weight, and an extension of the tumor doubling time. Mechanistically, the treatment induced tumor apoptosis and decreased cell proliferation. Finally, although doxorubicin caused the increase of fibrosis markers and oxidative stress in animal hearts, loading doxorubicin into nanobubbles avoided these effects preventing heart damage. The improvement of doxorubicin anti-tumor effects together with the prevention of heart damage suggests that the combination of doxorubicin-loaded nanobubbles with extracorporeal shock waves might be a promising drug delivery system for anaplastic thyroid cancer treatment.
\end{abstract}

\author{
Key Words \\ - extracorporeal shock waves \\ - nanobubbles \\ - doxorubicin \\ - anaplastic thyroid cancer \\ - drug delivery
}

Endocrine-Related Cancer (2017) 24, 275-286

\section{Introduction}

Anaplastic thyroid cancer (ATC) is one of the most lethal cancers, its median survival being only 5 months, and 1-year survival being less than 20\% (Smallridge \&
Copland 2010). To date, a standard therapy for ATC does not exist. The American Thyroid Association (Smallridge et al. 2012) and the National Comprehensive

Published by Bioscientifica Ltd 
Cancer Network guidelines (Haddad et al. 2015) suggest a multimodal approach including extensive resection followed by adjuvant chemo-radiotherapy. Nevertheless, the management of ATC remains a challenge, and ATC is still an incurable disease. Doxorubicin is the only cytotoxic drug approved for the treatment in monotherapy of ATC (Haddad et al. 2015). Unfortunately, it has a response rate below 22\% (Giuffrida et al. 2000) and, if not specifically targeted to the tumor cells, it causes severe side effects, among which cardiotoxicity is the most prominent. Notably, the severity of cardiac side effects and their occurrence are dose dependent (Minotti et al. 2004, Chatterjee at al. 2010, Zhang et al. 2012). Therefore, there is an increasing attention toward the development of new doxorubicin formulations to specifically deliver the drug to the tumor site increasing its anti-tumor efficacy and reducing its toxicity. To this aim, many formulations of doxorubicin-loaded nanoparticles have been developed (Wohlfart et al. 2011, Barenholz 2012, Golla et al. 2013, Yu et al. 2014, Razavi-Azarkhiavi et al. 2016). In general, nanoparticles can carry loaded drugs to the tumor site through the blood stream taking advantage of the enhanced permeability and retention effect, due to the defective vascular architecture of the tumor (Fang et al. 2011). However, the universal role and advantages of enhanced permeability and retention effect in cancer therapy are still debated. In fact, vessel leakage, the absence of a functional lymphatic system and increased extracellular matrix frictional resistance increase tumor interstitial fluid pressure and ultimately provoke disruption in blood flow directions, limiting drug delivery (Carmeliet and Jain 2011, Azzi et al. 2013). To overcome this problem, a better drug delivery to cancer tissues can be obtained by combining physical triggers (e.g. ultrasound (US)) to gas-cored micro and nanobubbles (NBs) (Gao et al. 2008, Collis at al. 2010, Cavalli et al. 2012, Cavalli et al. 2016). Indeed, US causes bubble cavitation resulting in cell sonoporation and allowing the extravasation of molecules (Collis et al 2010). Extracorporeal shock waves (ESWs) are short-duration focused acoustic waves widely used in urology for lithotripsy (Rassweiler et al. 2011) and, more recently, in several musculoskeletal diseases (Wang 2012). ESWs can be focused with high precision in depth, and they determine permeabilization of plasma membranes (Lauer et al. 1997, Kodama et al. 2002, Frairia et al. 2003, Catalano et al. 2007). As a consequence of ESWinduced cell permeability, ESWs increase the cytotoxic effects of different anti-cancer drugs (Frairia et al. 2003, Palmero et al. 2006, Catalano et al. 2007, Canaparo et al. 2008). All these features make ESWs an ideal tool to be used in combination with drug-loaded NBs. In line with this hypothesis, we recently demonstrated that new perfluoropentane-cored glycol chitosan NBs loaded with doxorubicin released the drug in response to ESW treatment in stabilized human ATC cell lines, resulting in increased intracellular drug content and enhanced doxorubicin cytotoxicity (Marano et al. 2016). Aim of the present study was to evaluate the in vivo efficacy of combining these doxorubicin-loaded NBs and ESWs in an ATC xenograft model with the final intent to improve doxorubicin anti-tumor effects and to reduce doxorubicininduced cardiotoxicity.

\section{Materials and methods}

\section{Nanobubble formulations}

Empty and doxorubicin-loaded perfluoropentane-cored glycol chitosan NBs were prepared by purposely tuning the method reported elsewhere (Marano et al. 2016) reaching a final amount of incorporated drug up to $150 \mathrm{~g} / \mathrm{mL}$. Physico-chemical characterization, loading capacity and encapsulation efficiency of nanobubbles were determined as described in the previous study (Marano et al. 2016).

\section{Cell line}

Stabilized ATC cell line, CAL-62, was purchased from Deutsche Sammlung von Mikroorganismen and Zellkulturen (Braunschweig, Germany), which certifies the origin and identity of the cells. Cells were routinely maintained in $75 \mathrm{~cm}^{2}$ flasks at $37^{\circ} \mathrm{C}$, in $5 \% \mathrm{CO}_{2}$ and $95 \%$ humidity, with $100 \mathrm{IU} / \mathrm{mL}$ penicillin and $100 \mu \mathrm{g} / \mathrm{mL}$ streptomycin (Gibco, Life Technologies Corp., Grand Island, NY, USA) added in DMEM-F12 (Invitrogen) supplemented with 10\% FCS (Euroclone, Wetherby, West Yorks, UK). CAL-62 cells are included in the list of the authenticated unique thyroid cancer cell lines (Schweppe et al. 2008).

\section{Xenograft model}

The study was conducted on Mus musculus in accordance with the national guidelines for the care and use of research animals and was approved by the Ethics Committee of the University of Turin and by the Italian Board of Health with authorization n.492/2015-PR. Suspension of $5 \times 10^{5}$ CAL-62 cells in Matrigel (1:1) (Corning) was injected under the skin in the two flanks of 7-week-old female NOD Scid Gamma (NSG) mice (Molecular Biotechnology

Published by Bioscientifica Ltd 
Center, Turin, Italy). When tumors reached a minimum volume of $100 \mathrm{~mm}^{3}$, animals were randomly assigned to different treatment groups.

To determine the time needed to deliver NBs to the tumors, $0.75 \mathrm{mg} / \mathrm{kg}$ doxorubicin-loaded NBs were intravenously (i.v.) injected in the animal tail vein. After $0.5,1.5,5$ and $24 \mathrm{~h}$, mice ( $n=5$ per group) were killed and doxorubicin was quantified in tumors by highperformance liquid chromatography (HPLC) as described below (doxorubicin quantification paragraph).

For all the other in vivo experiments, $100 \mu \mathrm{L}$ of $0.9 \%(\mathrm{v} / \mathrm{v})$ saline solution (CTRL; $n=5)$, or $0.75 \mathrm{mg} / \mathrm{kg}$ doxorubicin solution (DOXO; $n=5$ ), or $0.75 \mathrm{mg} / \mathrm{kg}$ doxorubicin-loaded glycol chitosan NBs (NBs-DOXO; $n=5$ ) were i.v. injected into the tail vein.

At the selected time, each mouse was anesthetized by intramuscular injection with $80 \mathrm{mg} / \mathrm{kg}$ tiletamine/ zolazepam (Zoletil 100, Virbac, France) and $16 \mathrm{mg} / \mathrm{kg}$ xylazine (Rompun, Bayer SpA, Germany) and treated with ESWs $\left(0.59 \mathrm{~mJ} / \mathrm{mm}^{2}, 500\right.$ pulses $)$ on one of the two tumors. A piezoelectric shock wave generator (Piezoson 100, Richard Wolf, Knittlingen, Germany), provided by Med \& Sport 2000 S.r.l. (Torino, Italy), was used for the study. This device generates focused underwater shock waves at various frequencies (1-4 pulses/s) and intensities $\left(0.05-1.48 \mathrm{~mJ} / \mathrm{mm}^{2}\right)$. Shock wave intensity is the energy at the focal point, defined as energy flux density (EFD) per impulse recorded as joules per area $\left(\mathrm{mJ} / \mathrm{mm}^{2}\right)$. The focus volume is defined as the zone where $50 \%$ of the maximum energy is delivered; with regard to the Piezoson 100, the focus zone has a length of $10 \mathrm{~mm}$ in the direction of the axis of the shock wave propagation and a diameter of $2.5 \mathrm{~mm}$ perpendicular to this axis. For treating mice, the hair over the tumor was shaved, and ultrasound gel was applied to the naked skin. The transducer was placed in close contact with the tumor. The position and the angle of the mouse were adjusted to locate the tumor at the focal spot and, thus, allow the focused wave to propagate throughout the cancer.

Treatments were repeated once a week for a total duration of 21 days. Tumor volume was assessed every seven days. Tumors were measured with calipers, and volumes were calculated with the formula $\mathrm{a}^{2} \times \mathrm{b} \times 0.5$, where $\mathrm{a}$ is the shortest diameter and $\mathrm{b}$ is the diameter perpendicular to a. Doubling time of tumor volume was calculated according the following formula: $\mathrm{T} \times \log _{2} /$ $\left(\log V_{f}-\log V_{i}\right)$, where $V_{f}$ is the final tumor volume, $V_{i}$ is the initial tumor volume and $\mathrm{T}$ is the time interval (days) between $V_{f}$ and $V_{i}$ (Guo et al. 2009).
After the mice were killed, tumors and hearts were collected. Tumors were weighed and photographed by digital camera. Pieces of tumors and hearts were either fixed in $10 \%$ buffered formalin, or frozen, or put in All Protect Tissue Reagent (Qiagen).

\section{Doxorubicin quantification}

Tissue extracts from frozen tumors and hearts were prepared by adding one volume of methanol followed by two volumes of $1 \mathrm{M}$ Tris buffer with $\mathrm{pH}$ 8.5. The mixtures were homogenized using Polytron (Kinematica GMBH, Eschbach, Germany), and the tissue homogenates were kept on ice for $15 \mathrm{~min}$ before adding seven volumes of acetonitrile. The mixtures were vortexed and allowed to stand at room temperature for $15 \mathrm{~min}$ before removing the precipitated proteins by centrifugation at $3000 \mathrm{~g}$ for $5 \mathrm{~min}$. After centrifugation, clear supernatants were assessed for doxorubicin content by HPLC. HPLC system consisted of a pump (LC-9A PUMP C, Shimadzu, Japan) equipped with a fluorescence detector (Chrompack, Japan) and analyses were performed using an Agilent TC C18 column $(250 \mathrm{~mm} \times 4.6 \mathrm{~mm}, 5 \mu \mathrm{m})$. To calculate the drug concentration, a linear calibration curve was set up with a concentration range of $0.025-2.5 \mathrm{~g} / \mathrm{mL}$ with a regression coefficient of 0.999 .

\section{Tunel assay}

Apoptosis was evaluated by TUNEL (terminal deoxynucleotidyl transferase-mediated dUTP nick end labeling) assay (ApopTag Plus, Millipore). Briefly, paraffin embedded slices of tumors were deparaffined and TUNEL assay was performed following the manufacturer's instruction. Positive cells were stained by a brown precipitate of oxidized 3,3'-diaminobenzidine (DAB) (Dako, Agilent Technologies). Slides were observed under optical microscope Olympus AX70 PROVIS (Olympus). To count positive cells, pictures were acquired by digital camera suite Leica Application Suite X 2.0.0.14332 (Leica Microsystems, Wetzlar, Germany). After excluding areas of necrosis, the number of apoptotic cells per field was counted in 10 randomly chosen sections using $\times 200$ final magnification.

\section{Proliferating cell nuclear antigen (PCNA)}

Tumor paraffin sections were deparaffined and probed using an anti-PCNA antibody (1:300 dilution in PBS 0.1\%

Published by Bioscientifica Ltd 
BSA; Santa Cruz Biotechnology). Secondary antibody was a goat anti-mouse horseradish peroxidase (HPR)-conjugated antibody (1:300 dilution in PBS 0.1\% BSA; Thermo Fisher Scientific). DAB (Dako, Agilent Technologies) was used as substrate of HPR conjugated to secondary antibody. To count PCNA-positive cells, pictures were acquired by digital camera suite Leica Application Suite X 2.0.0.14332 (Leica Microsystems). The ten fields with the highest density of positive nuclei were captured at $\times 200$ final magnification and positive vs total cells were counted using ImageJ software. Only nuclei with a strongly positive label were counted.

\section{Hematoxylin and Eosin stain}

Heart paraffin sections were deparaffined and stained with hematoxylin and eosin. Scansions of the entire slides were captured by Aperio ScanScope slide scanner (Leica Microsystems).

\section{Masson's trichrome stain}

Collagen fibers in hearts were detected by Masson's trichrome stain kit (Bio-Optica Milano S.p.a., Milan, Italy). Briefly, paraffin-embedded slices of hearts were deparaffined and trichrome stain was performed following the manufacturer's instruction. Collagen fibers were stained by aniline blue solution included in the kit. Slides were observed under optical microscope Olympus AX70 PROVIS (Olympus). Pictures were acquired by digital camera suite Leica Application Suite X 2.0.0.14332 (Leica Microsystems). The percentage of blue area vs total area was counted in ten randomly chosen fields at $\times 200$ final magnification using ImageJ software.

\section{Gene expression}

Total RNA was extracted from heart pieces, homogenizing tissues by Polytron (Kinematica GMBH) in TRIzol Reagent (Invitrogen). DNase I was added to remove the remaining genomic DNA. $1 \mu \mathrm{g}$ total RNA was reverse-transcribed with iScript cDNA Synthesis Kit (BioRad Laboratories), following the manufacturer's protocol.

Primers (Table 2) were designed using Beacon Designer 5.0 software according to parameters outlined in the BioRad iCycler Manual. Specificity of primers was confirmed by BLAST analysis. Real-time PCR was performed using a
BioRad iQ iCycler Detection System (BioRad Laboratories) with SYBR green fluorophore. Reactions were performed in a total volume of $25 \mu \mathrm{L}$ including $12.5 \mu \mathrm{L}$ IQ SYBR Green Supermix (BioRad Laboratories), $1 \mu \mathrm{L}$ of each primer at $10 \mu \mathrm{M}$ concentration and $5 \mu \mathrm{L}$ of the previously reverse-transcribed cDNA template. Protocol for primer set up was optimized using seven serial $5 \mathrm{X}$ dilutions of template cDNA obtained from cells in basal conditions. The protocol used is as follows: denaturation $\left(95^{\circ} \mathrm{C}\right.$ for $5 \mathrm{~min})$, amplification repeated 40 times $\left(95^{\circ} \mathrm{C}\right.$ for $15 \mathrm{~s}$ and $60^{\circ} \mathrm{C}$ for $30 \mathrm{~s}$ ). A melting curve analysis was performed following every run to ensure a single amplified product for every reaction. All reactions were carried out at least in triplicate for each sample. Results were normalized using the geometric mean for three different housekeeping genes (ribosomal protein L13A, ribosomal protein large P0, and glyceraldehyde-3-phosphate dehydrogenase) and expressed as relative expression fold vs untreated controls.

\section{Protein extraction}

Total proteins were extracted from heart tissue homogenates in 10\% (w/v) RIPA buffer (0.5\% Nonidet P-40, 0.5\% sodium deoxycholate, $0.1 \%$ SDS, $10 \mathrm{mmol} / \mathrm{L}$ EDTA and protease inhibitors). After $40 \mathrm{~min}$ of incubation in ice, samples were sonicated and cleared by centrifugation at $14,000 \mathrm{~g}$ at $4^{\circ} \mathrm{C}$ for $20 \mathrm{~min}$. Supernatants were used for ROS and glutathione determinations and for immunoblotting.

\section{Reactive oxygen species (ROS)}

ROS were measured using the $2^{\prime}, 7^{\prime}$-dichlorofluorescein diacetate (DCFH-DA) probe. DCFH-DA is a stable, nonfluorescent molecule, that is hydrolyzed by esterases to non-fluorescent 2',7'-dichlorofluorescein (DCFH), which is rapidly oxidized in the presence of peroxides to the highly fluorescent $2^{\prime}, 7^{\prime}$-dichlorofluorescein (DCF). Protein extracts were incubated with $5 \mu \mathrm{M}$ of DCFH-DA for $15 \mathrm{~min}$ at $37^{\circ} \mathrm{C}$, and then, DCF was measured fluorimetrically at $485 \mathrm{~nm}$ excitation and $538 \mathrm{~nm}$ emission.

\section{Oxidized-to-reduced glutathione ratio (GSSG/GSH)}

GSSG/GSH was assessed by mixing in a cuvette $0.05 \mathrm{M}$ Na-phosphate buffer (pH 7.0), $1 \mathrm{mM}$ EDTA (pH 7.0) and $10 \mathrm{mM}$ dithionitrobenzoic acid (Sigma-Aldrich) plus an aliquot of the sample. After 2 min of reaction, 
Table 1 Physico-chemical characteristics of NB formulations.

\begin{tabular}{|c|c|c|}
\hline & Empty NBs & Doxorubicin-loaded NBs \\
\hline Average diameters \pm S.D. $(\mathrm{nm})$ & $344.6 \pm 13.8$ & $356.2 \pm 15.1$ \\
\hline PDI (Polydispersity Index) & $0.19 \pm 0.01$ & $0.20 \pm 0.02$ \\
\hline$\zeta$-Potential \pm S.D. $(\mathrm{mV})$ & $29.8 \pm 2.122$ & $30.4 \pm 2.85$ \\
\hline Encapsulation efficiency & - & $75 \%$ \\
\hline Loading capacity & - & $4.5 \%$ \\
\hline
\end{tabular}

GSH content was evaluated by reading absorbance at $412 \mathrm{~nm}$, calculated referring to a standard curve and expressed as $\mu \mathrm{g} / \mathrm{mg}$ protein. Suitable volumes of diluted glutathione reductase and of reduced nicotinamide adenine dinucleotide phosphate were then added to convert the oxidized glutathione to the reduced form and then evaluate the total glutathione level. The difference between total glutathione and GSH content represents the GSSG content, also expressed as $\mu \mathrm{g} / \mathrm{mg}$ protein.

\section{Immunoblotting}

SDS-PAGE was performed on $12 \%$ acrylamide (SigmaAldrich) gel, loading $30 \mu \mathrm{g}$ protein/well. Separated proteins were electro-transferred onto PVDF membrane (BioRad Laboratories, Inc.) and probed with anti-manganese superoxide dismutase (Mn-SOD) antibody (1:1500 dilution, Upstate, Millipore) and with anti- $\alpha$-tubulin antibody (1:100 dilution, Abcam, UK) to check protein loading. Proteins were detected with Pierce Super Signal chemiluminescent substrate following the manufacturer's instructions. Bands were photographed and analyzed using ImageJ software.

\section{Statistical analysis}

Data are expressed throughout the text as means \pm S.D., calculated from at least three different experiments. Comparison between groups was performed with analysis of variance (one-way ANOVA), and the threshold of significance was calculated with the Bonferroni test. Statistical significance was set at $P<0.05$.

\section{Results}

\section{Physico-chemical characteristics of nanobubble formulations}

Doxorubicin-loaded NBs showed an average diameter of about $350 \mathrm{~nm}$ and positive surface charge (Table 1). A prolonged drug release kinetics of doxorubicin from doxorubicin-loaded nanobubbles was demonstrated in in vitro-release studies, as previously reported (Marano et al. 2016).

In particular, less than $10 \%$ of the drug was released from nanobubbles and no initial burst effect was observed, after $6 \mathrm{~h}$ in the absence of ESWs. On the contrary, following ESW treatment, doxorubicin was released in a larger extent from nanobubbles.

\section{Anti-tumor effects}

In order to establish the time needed to deliver NBs to the tumor, doxorubicin content was measured in tumors of ATC-bearing mice at different times after doxorubicinloaded NB injection. As shown in Fig. 1A, after $30 \mathrm{~min}$, the drug was already present in the tumor tissue, and no significant difference in drug content was observed up to $24 \mathrm{~h}$. Therefore, 1.5 hours was chosen as the waiting time before ESW treatment as this time better fitted with our ESW treatment schedule.

Table 2 Primers for Real-time PCR.

Gene
-sma (alpha-smooth muscle actin)
Col1a1 (collagen type 1 alpha 1 )
RplpO (ribosomal protein L13a)
Gapdh (glyceraldehyde-3-phosphate dehydrogenase)

\section{Primers}

Sense: 5'-GCCAGTCGCTGTCAGGAACC-3'

Antisense: 5'-CAGAGCCCAGAGCCATTGTCG-3'

Sense: 5'-GCCACTGCCCTCCTGACG-3'

Antisense: 5'-AGATCAAGCATACCTCGGGTTTCC-3'

Sense: 5'-GTGGTCCCTGCTGCTCTCAAG-3'

Antisense: 5'-GCTGTCACTGCCTGGTACTTCC-3'

Sense: 5'-AGGAAGAGTCGGAGGAATCAGATG-3'

Antisense: 5'-CTTGGTTGCTTTGGCGGGATTAG-3'

Sense: 5'-AGCAAGGACACTGAGCAAGAGAG-3'

Antisense: 5'-GGGATGGAAATTGTGAGGGAGATG-3' 
To test the anti-tumor effect of different treatments, ATC-bearing mice were randomly divided into three groups: control mice (CTRL), doxorubicin-treated mice (DOXO) and mice treated with doxorubicin-loaded NBs (NBs-DOXO). After $1.5 \mathrm{~h}$, one of the two tumors of each animal was treated with ESWs $\left(0.59 \mathrm{~mJ} / \mathrm{mm}^{2}\right.$, 500 pulses), and treatments were repeated once a week for a 21-day period. Treatments are schematized in Fig. 1B.

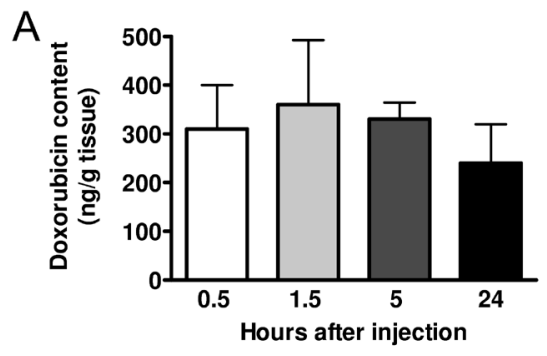

C

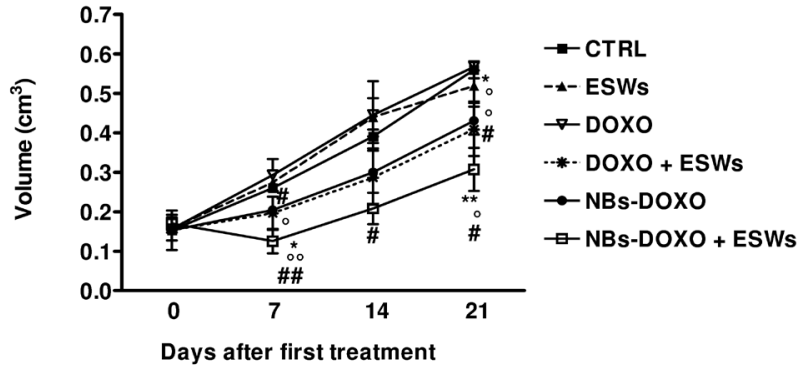

$E$

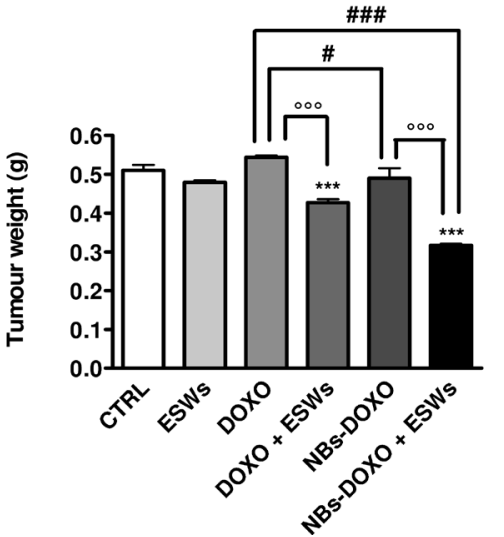

As shown in Fig. 1C, after 21 days, tumor volume was significantly reduced in mice that received the combined treatment with NBs-DOXO and ESWs with respect to CTRL $(P<0.01)$, DOXO $(P<0.05)$ and NBs-DOXO $(P<0.05)$ groups. The significant increase of the tumor doubling time (Table 3) after the combined treatment (vs CTRL, $P<0.001$; vs DOXO, $P<0.001$; vs NBs-DOXO, $P<0.01)$ was in agreement with the significant decrease of tumor volume. The most efficient anti-tumor effect of
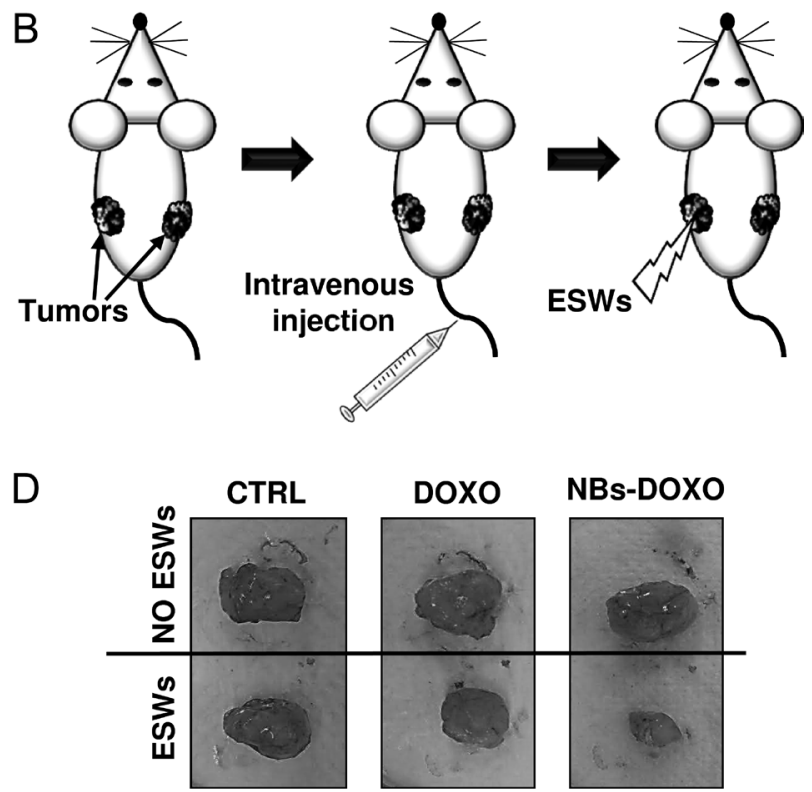

$\mathrm{F}$

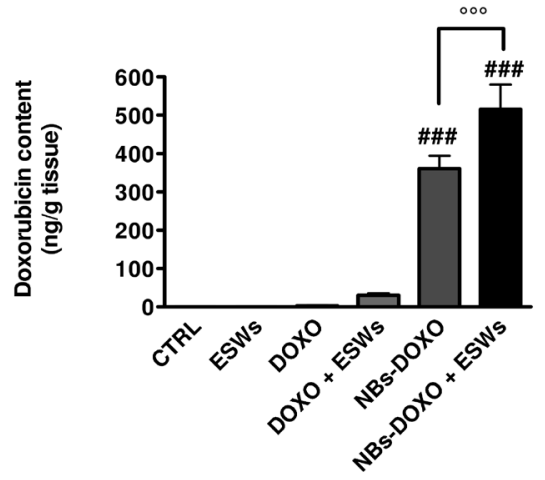

Figure 1

Tumor growth inhibition. (A) Doxorubicin content at $0.5,1.5,5$ and $24 \mathrm{~h}$ after i.v. injection of doxorubicin-loaded NBs ( $n=5$ per group) expressed as ng of drug/g tissue. (B) Schematic representation of the treatments of ATC-bearing mice. Two tumors per mouse were allowed to grow in the flanks of 7-week-old female NSG mice. When tumors reached an appropriate volume $\left(100 \mathrm{~mm}^{3}\right), 100 \mu \mathrm{L}$ vehicle, or $0.75 \mathrm{mg} / \mathrm{kg}$ doxorubicin solution, or $0.75 \mathrm{mg} / \mathrm{kg}$ doxorubicin-loaded NBs were i.v. injected into the tail vein. After 1.5 h, one tumor for each mouse was treated with ESWs. (C) Tumor volumes at $0,7,14$ and 21 days ( $n=5$ per group), calculated with the formula $\mathrm{a}^{2} \times \mathrm{b} \times 0.5$, where $\mathrm{a}$ is the shortest diameter and $\mathrm{b}$ is the diameter perpendicular to $\mathrm{a}$. (D) Representative pictures of tumors after 21 days of treatment. (E) Weights of tumors at 21 days ( $n=5$ per group). (F) Doxorubicin content ( $n=5$ per group) after 21 days of treatments expressed as $\mathrm{ng}$ of drug/g tissue. Significances were calculated with one-way ANOVA analysis and Bonferroni

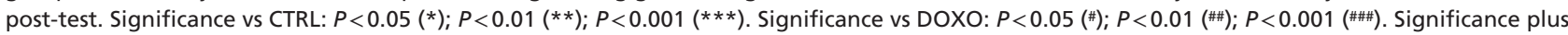
ESWs vs no ESWs: $P<0.05\left({ }^{\circ}\right) ; P<0.01\left({ }^{\circ}\right) ; P<0.001\left({ }^{\circ 00}\right)$. 
the combined treatment was already evident by visual observation of tumors (Fig. 1D). Moreover, the combined treatment determined the greatest reduction in tumor weights (vs CTRL, DOXO and NBs-DOXO, $P<0.001$ ) as demonstrated in Fig. 1E.

In accordance with these observations, the highest doxorubicin content (Fig. 1F) was found in tumors treated with doxorubicin-loaded NBs plus ESWs (vs DOXO, $P<0.001$; vs NBs-DOXO, $P<0.001$ ).

To explore the mechanisms underlying the tumor growth inhibition, we evaluated apoptotic (Fig. 2A and B) and proliferating cells (Fig. 2C and D). As shown in Fig. 2B, the number of apoptotic cells in tumors treated with NBs-DOXO was significantly increased with respect to CTRL $(P<0.001)$ and to DOXO $(P<0.01)$ groups. However, in tumours treated with the combination of NBs-DOXO and ESWs, the greatest number of apoptotic cells was observed (vs CTRL, $P<0.001$; vs DOXO, $P<0.001$; vs NBs-DOXO, $P<0.01)$.

Moreover, the combined treatment with NBs-DOXO and ESWs decreased PCNA-positive cells with respect to any other condition (Fig. 2C). Fig. 2D shows the percentage of PCNA-positive cells. In tumors treated with NBs-DOXO, the percentage was significantly reduced with respect to CTRL $(P<0.001)$ and to DOXO $(P<0.01)$ groups. The combined treatment elicited the greatest reduction in the percentage of proliferating cells (vs CTRL, $P<0.001$; vs DOXO, $P<0.001$; vs NBs-DOXO, $P<0.01)$.

\section{Cardiotoxicity}

Hearts of doxorubicin-treated mice showed areas of disorganized muscle fibers with poor cellularity (Fig. 3A). On the contrary, cardiac tissue of mice treated with NBsDOXO conserved the same appearance of hearts of the CTRL group (Fig. 3A).

Table 3 Doubling time ${ }^{1}$ of tumor volume.

\begin{tabular}{lc}
\hline Treatment & Doubling time (Days) \\
\hline CTRL & $11.2 \pm 0.4$ \\
ESWs & $12.3 \pm 1$ \\
DOXO & $11.2 \pm 0.2$ \\
DOXO +ESWs & $14.8 \pm 2.4$ \\
NBs-DOXO & $14 \pm 1.3$ \\
NBs-DOXO +ESWs & $24.5 \pm 4.3(* * *)(\# \#)\left({ }^{\circ \circ}\right)^{2}$ \\
\hline
\end{tabular}

'Doubling time of tumor volume was estimated according to the formula described by Guo et al. (2009) and reported in the Materials and methods section.

2 Significances were calculated with one-way ANOVA analysis and Bonferroni post-test. Significance vs CTRL: $P<0.001(* * *)$. Significance vs DOXO: $P<0.001$ (\#\#). Significance plus ESWs vs no ESWs: $P<0.01\left({ }^{\circ}{ }^{\circ}\right)$.
As collagen is usually found in fibrotic tissues (Hinz 2007), Masson's trichrome specific stain was performed. Extended collagen blue areas were observable in hearts of DOXO group, while they were present in a very little extension in CTRL and in NBs-DOXO groups (Fig. 3B). Indeed, the percentage of collagen areas in heart of DOXO group was significantly increased compared to that in CTRL group $(P<0.001$, Fig. 3C). On the contrary, the percentage of collagen in hearts of NBs-DOXO group was significantly reduced with respect to DOXO group $(P<0.001$, Fig. $3 \mathrm{C})$ and was comparable to that of the CTRL group.

Moreover, doxorubicin significantly increased the expression of collagen type 1 alpha 1 (Col1a1; $P<0.05$, Fig. 3D) and of alpha-smooth muscle actin ( $\alpha$-sma; $P<0.001$, Fig. 3E) genes, well-established markers of cardiac fibrosis (Hinz 2007). Encapsulating the drug into NBs counteracted these increases and resulted in Col1a1 and $\alpha$-sma expression levels similar to those of the CTRL group $(P<0.05$ for Col1a1, Fig. 3D; $P<0.001$ for $\alpha$-sma, Fig. 3E).

Being an index of doxorubicin-induced heart damage (Singal et al. 2000, Spallarossa et al. 2006, Mukhopadhyay et al. 2009, Zhao et al. 2010), oxidative stress was also assessed. Doxorubicin significantly induced cardiac ROS production (vs CTRL, $P<0.001$, Fig. 4A), increased GSSG/GSH ratio (vs CTRL, $P<0.01$, Fig. 4B) and Mn-SOD protein expression (vs CTRL, $P<0.05$, Fig. $4 \mathrm{C}$ and D). Notably, in hearts of NBs-DOXO-treated mice, ROS levels, GSSG/GSH ratio and Mn-SOD were significantly reduced with respect to DOXO group $(P<0.001$, Fig. 4A; $P<0.05$, Fig. 4B; $P<0.05$, Fig. $4 \mathrm{C}$ and $\mathrm{D}$ ), and comparable to those of the CTRL group. In accordance with these observations, doxorubicin was detected only in hearts of DOXO group, whereas it was undetectable in the hearts of NBs-DOXO group (Fig. 4E).

\section{Discussion}

The present in vivo preclinical study demonstrates that in ATC, a lethal tumor with no standard therapy, the combined treatment with doxorubicin-loaded glycol chitosan NBs and ESWs enhances the anti-tumor effects of doxorubicin and, at the same time, prevents the druginduced heart damage.

Doxorubicin delivery to the tumor cells, with the specific aim to increase its anti-tumor efficacy and to reduce its side effects, is an important goal in ATC therapy. In fact, even if doxorubicin is the only chemotherapeutic drug approved for ATC treatment in monotherapy (Haddad et al. 2015), it has a poor clinical response (Giuffrida \& Gharib 2000), and it causes severe

Published by Bioscientifica Ltd 
A

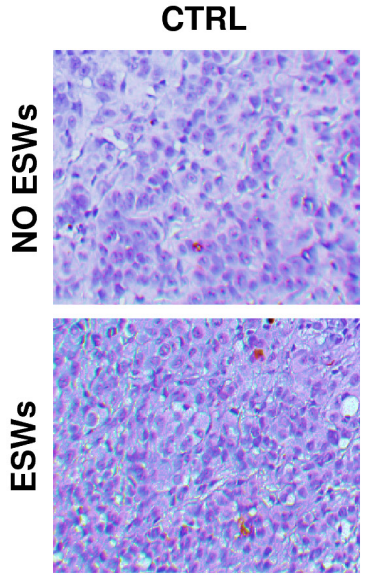

C

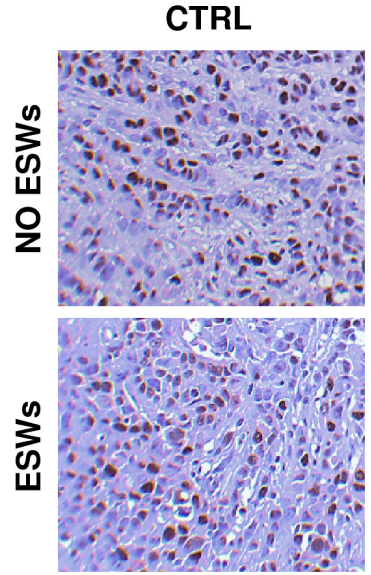

DOXO

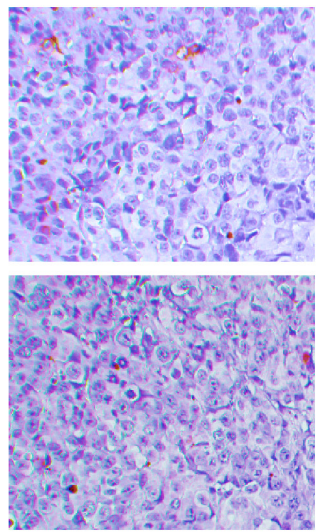

DOXO

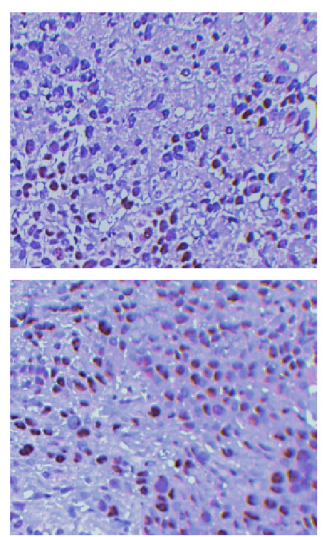

NBs-DOXO

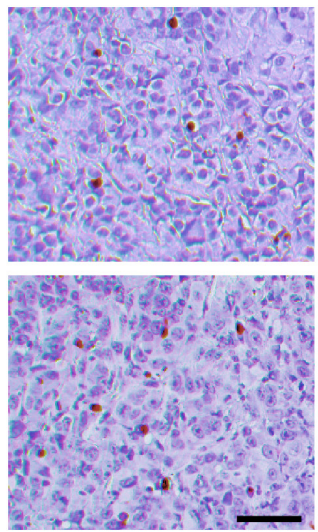

NBs-DOXO

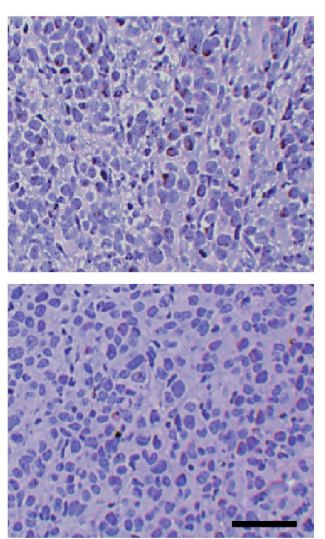

B

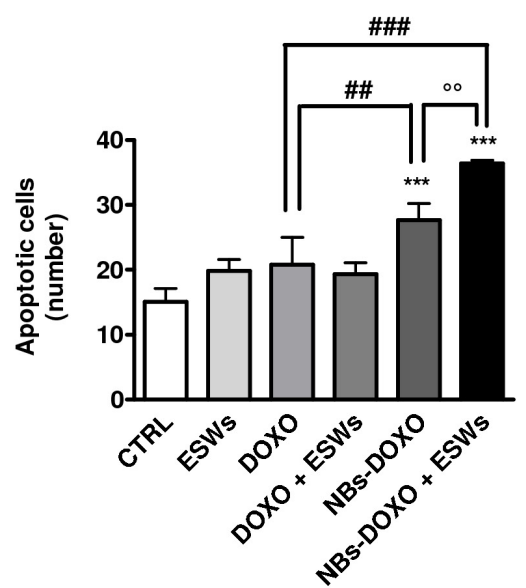

D

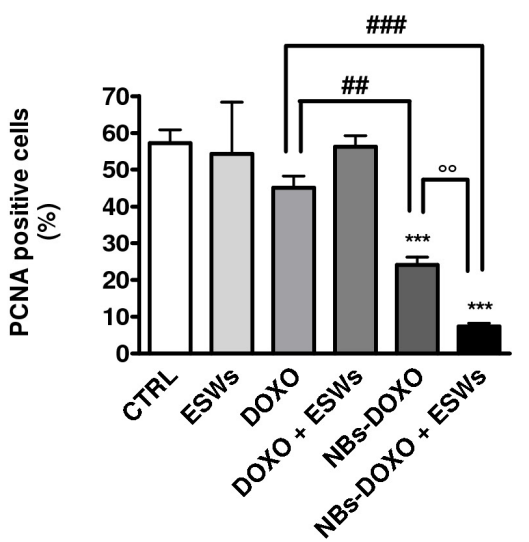

Figure 2

Apoptosis and proliferation of tumor cells. (A) Representative pictures of TUNEL stained slides after 21 days of treatments (scale bar: $50 \mu \mathrm{m}$ ). (B) Quantification of apoptotic cells per field, excluding necrosis areas, by ImageJ software in 10 randomly chosen sections at $\times 200$ final magnification ( $n=5$ per group). (C) Representative pictures of PCNA slides after 21 days of treatments (scale bar: $50 \mu \mathrm{m}$ ). (D) Percentage of PCNA-positive cells per field quantified by ImageJ software counting 10 randomly chosen $\times 200$ final magnification ( $n=5$ per group). Significances were calculated with one-way ANOVA analysis and Bonferroni post-test. Significance vs CTRL, $P<0.001(* * *)$; significance vs DOXO, $P<0.01$ (\#\#), $P<0.001$ (\#\#\#); significance plus ESWs vs no ESWs, $P<0.01\left({ }^{\circ}\right)$. A full colour version of this figure is available at http://dx.doi.org/10.1530/ERC-17-0045.

dose-dependent side effects among which cardiotoxicity is the most prominent (Minotti et al. 2004, Chatterjee et al. 2010, Zhang et al. 2012).

Based on the promising results of our recent in vitro study (Marano et al. 2016), where we delineated the cytotoxic effects and the mechanism of this new combined treatment with NBs-DOXO and ESWs, we here go further moving to a xenograft in vivo model. Even if the cytotoxic efficacy of different types of nanoparticles to deliver doxorubicin has been reported in other tumor histotypes (Du et al. 2011, Wohlfart et al. 2011, Barenholz 2012, Golla et al. 2013, Yu et al. 2014, Fan et al. 2016, Lin et al. 2016, Razavi-Azarkhiavi et al. 2016); to date, no other study has used delivery systems to target doxorubicin in ATC. In 2011, Du and coworkers showed that US-responsive doxorubicin-loaded PEGylated perfluoropentane NBs resulted in a more efficient inhibition of tumor growth in hepatocarcinoma-bearing mice (Du et al. 2011). Others reported a greater anti-tumor efficacy in human prostate cancer xenograft model using doxorubicin-loaded lipid NBs in combination with US (Fan et al. 2016). In nude mice xenograft of fibrosarcoma, the combination of US with asparagine-glycine-arginine (NGR) peptide modified NBs, loaded with doxorubicin conjugated with cell-permeable peptides, resulted in higher tumor growth inhibition with respect to NBs or doxorubicin alone (Lin et al. 2016).

In 2012, Cavalli and coworkers developed stable chitosan NBs able to release DNA into COS7 cells after US treatment. In the presence of US, the perfluoropentane of the nanobubble core, which is liquid at room temperature, underwent a conversion from droplet to bubble at $37^{\circ} \mathrm{C}$, inducing DNA release into the cells (Cavalli et al. 2012).

Published by Bioscientifica Ltd 
A

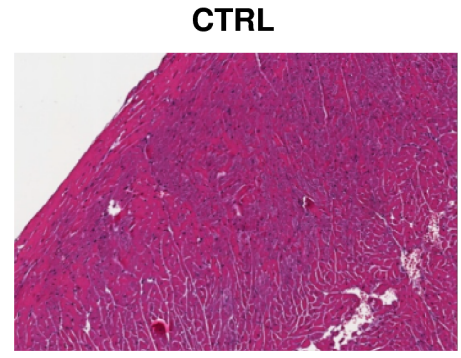

B

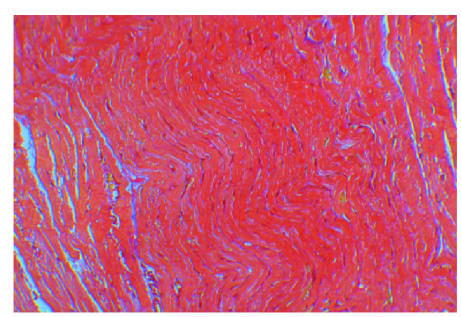

C

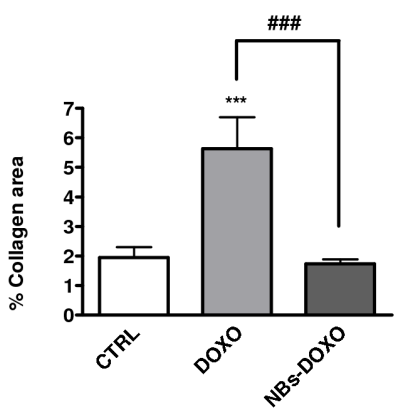

DOXO

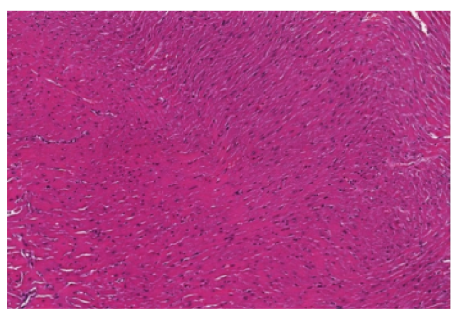

DOXO

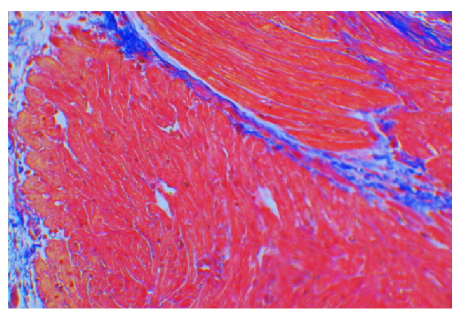

D

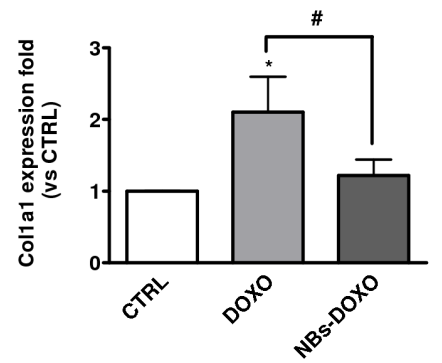

NBs-DOXO

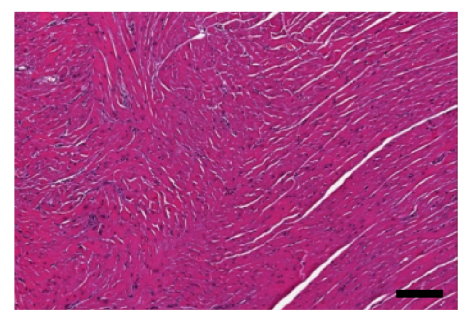

NBs-DOXO

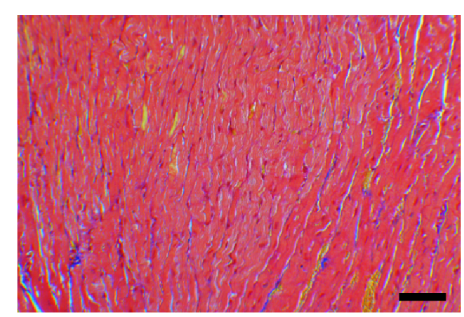

E

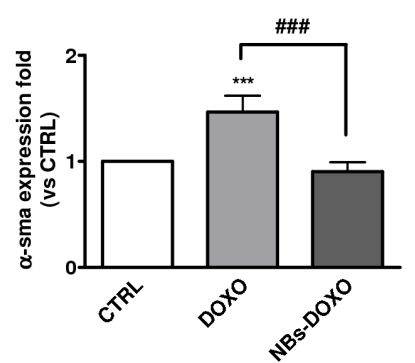

Figure 3

Heart fibrosis. (A) Representative pictures of Hematoxylin-Eosin-stained heart slide scans (scale bar: $100 \mu \mathrm{m} ; n=5$ per group). (B) Representative pictures of Masson's trichrome-stained heart slides for collagen identification (scale bar: $50 \mu \mathrm{m} ; n=5$ per group). (C) Percentage of blue area, corresponding to collagen fibers, vs total area counted on ten randomly chosen fields at $\times 200$ magnification by ImageJ software. (D) Cardiac gene expression of col1a 1 and (E) $\alpha$-sma evaluated by RT-PCR ( $n=5$ per group). Results are normalized vs three different housekeeping genes (ribosomal protein L13A, ribosomal protein large PO, and glyceraldehyde-3-phosphate dehydrogenase) and expressed as relative fold expression vs the CTRL group. Significances were calculated with one-way ANOVA analysis and Bonferroni post-test. Significance vs CTRL, $P<0.05(*), P<0.001(* * *)$; significance vs DOXO, $P<0.05(*)$, $P<0.001$ (\#\#). A full colour version of this figure is available at http://dx.doi.org/10.1530/ERC-17-0045.

The US-responsive doxorubicin-loaded nanoparticulate system prepared in 2011 by Du and coworkers was based on the same mechanism (nanodroplet/NB transition). Indeed, PEGylated perfluoropentane nanodroplets were converted into $\mathrm{NBs}$ at $37^{\circ} \mathrm{C}$, and only little drug was released if no US was applied (Du et al. 2011).

The novelty of both our previous (Marano et al. 2016) and present works is not only the use of new perfluoropentane drug-loaded NBs, but it is especially the use of ESWs as novel physical strategy to trigger drug release from NBs, specifically at the tumor site. Indeed, unlike US, ESWs have no heating effects, and this characteristic could be an advantage for in vivo applications as temperature increase is difficult to control spatially and temporally, especially in large tumors with heterogeneous vascularization, such as ATC (Diederich \& Hynynen 1999).
In our xenograft murine model of human ATC, after doxorubicin-loaded NB injection in the tail vein, the drug was already detected in tumors after $30 \mathrm{~min}$, according to the previously mentioned studies where a 30-minute waiting time before US treatment has been reported (Du et al. 2011, Fan et al. 2016, Lin et al. 2016). As in our experimental conditions, the drug concentration remained stable up to $24 \mathrm{~h}$, we chose a 1.5-hour lag time before applying ESWs as this time better fitted with our ESW treatment schedule. At the end of the 21-day period, combining doxorubicinloaded NBs and ESWs elicited the best effect in terms of tumor growth inhibition, reducing tumor volumes and weights and extending the tumor doubling time. The greatest doxorubicin accumulation observed in tumors treated with drug-loaded NBs plus ESWs paralleled the antitumor effect. Present data further confirm our previous

Published by Bioscientifica Ltd. 

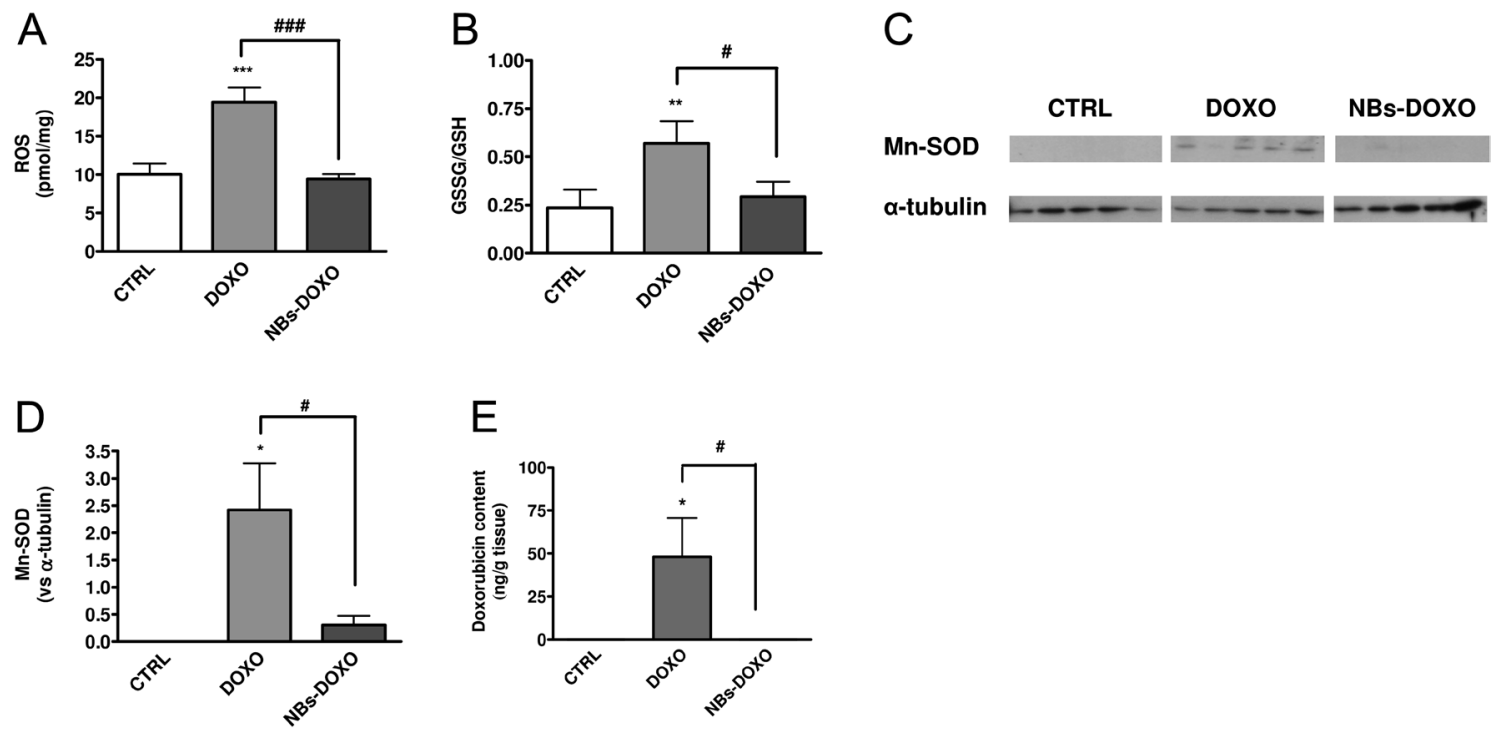

\section{Figure 4}

Oxidative stress in cardiac tissue. (A) ROS measured in heart tissue after 21 days of treatments ( $n=5$ per group). Results were expressed as pmol/mg protein content. (B) GSSG and GSH content in cardiac tissue after 21 days of treatments ( $n=5$ per group) expressed as ratio between GSSG and GSH content. (C) A typical Mn-SOD western blot on heart extracts is reported. (D) Western blot quantification by ImageJ software ( $n=5$ per group). (E) Doxorubicin content in hearts after 21 days of treatments ( $n=5$ per group) expressed as $n g / g$ tissue. Significances were calculated with one-way ANOVA analysis and Bonferroni post-test. Significance vs CTRL, $P<0.05(*), P<0.01(* *), P<0.001\left(^{* * *}\right)$; significance vs DOXO, $P<0.05(*), P<0.001(\# \#)$.

in vitro results, where the combination of ESWs with doxorubicin-loaded NBs elicited the greatest intracellular drug accumulation in ATC cells and significantly increased doxorubicin cytotoxicity, decreasing the growth inhibition fifty (the amount of drug that that inhibits $50 \%$ of cell growth) by about 40\% (Marano et al. 2016).

Furthermore, we here demonstrated that loading doxorubicin into NBs avoided doxorubicin-induced heart damage and oxidative stress. In fact, although doxorubicin-induced disorganization of muscle fibers, hearts of mice treated with doxorubicin-loaded NBs had a normal morphological aspect. Moreover, loading doxorubicin into NBs prevented the increase of the fibrosis markers Col1a1 and $\alpha$-sma, determined by free doxorubicin. Doxorubicin is a conventional anthracycline acting by intercalation with DNA base pairs (Minotti et al. 2004) or through inhibition of topoisomerase I and II by direct linking to the two enzymes (Tacar et al. 2013). ROS production is another mechanism of doxorubicin antitumor activity that causes direct damage to the DNA, RNA, lipids and proteins (Sinha et al. 1987). However, doxorubicin-induced cardiomyopathy is strongly linked to an increase in cardiac oxidative stress, and the induction of free radical production is the main mechanism through which doxorubicin injures the myocardium (Singal et al. 2000, Spallarossa et al. 2006, Mukhopadhyay et al. 2009, Zhao et al. 2010). Loading doxorubicin into NBs determined the inhibition of doxorubicin-induced ROS production and reduction of both GSSG/GSH ratio and Mn-SOD protein. ROS have an important role in the progression of fibrosis modulating fibroblast proliferation and differentiation into myofibroblasts expressing $\alpha$-SMA and producing extracellular matrix proteins, as type 1 collagen (Kuwahara et al. 2002, Krstić et al. 2015). The absence of the drug in hearts of mice treated with doxorubicin-loaded NBs explained the prevention of cardiac side effects. As the probability of developing cardiomyopathy is largely dose dependent (Sheppard et al. 2013), the possibility to use lower drug doses, together with delivering doxorubicin to the tumor tissue, is fundamental to reduce drug accumulation in non-target districts.

In summary, the drug delivery system presented in this study, by adding ESWs as physical trigger to glycol chitosan doxorubicin-loaded NBs, allowed increasing drug content in tumor tissue favoring the increase of doxorubicin anti-tumor effect with respect to the classic treatment with the free drug. Moreover, the use of glycol chitosan NBs, reduced doxorubicin accumulation in heart avoiding cardiac oxidative stress and fibrosis.

In conclusion, this preclinical study on the use of ESWs and doxorubicin-loaded NBs suggests that this combined treatment may be a promising drug delivery tool for precisely targeting doxorubicin to ATC. The possibility to focus ESWs with high precision in depth without heating effect, with consequent controlled drug release in the tumor tissue, makes this new strategy feasible

Published by Bioscientifica Ltd. 
for other aggressive solid tumors in which chemotherapy unfortunately remains the first option.

Given these promising results and the lack of a standard therapy for ATC, the further step will be clinical trials with the hope to open new perspectives for improving ATC treatment and patient quality of life.

\section{Declaration of interest}

The authors declare that there is no conflict of interest that could be perceived as prejudicing the impartiality of the research reported.

\section{Funding}

Fondazione CRT (grant number=RF2013.1289), Turin, Italy to MGC. "Research Fund ex-60\%", University of Turin, Turin, Italy to MGC and to RC.

\section{Acknowledgments}

The authors thank Massimo Cedrino, Federica Antico and Alessia Cento for technical support.

\section{References}

Azzi S, Hebda JK \& Gavard J 2013 Vascular permeability and drug delivery in cancers. Frontiers in Oncology 3 211. (doi:10.3389/ fonc.2013.00211)

Barenholz YC 2012 Doxil@-the first FDA-approved nano-drug: lessons learned. Journal of Controlled Release 160 117-134. (doi:10.1016/j. jconrel.2012.03.020)

Canaparo R, Serpe L, Zara GP, Chiarle R, Berta L \& Frairia R 2008 High energy shock waves (ESW) increase paclitaxel efficacy in a syngeneic model of breast cancer. Technology in Cancer Research \& Treatment $\mathbf{7}$ 117-124. (doi:10.7785/tcrt.2012.500182)

Carmeliet P \& Jain RK 2011 Principles and mechanisms of vessel normalization for cancer and other angiogenic diseases. Nature Reviews: Drug Discovery 10 417-427. (doi:10.1038/nrd3455)

Catalano MG, Costantino L, Fortunati N, Bosco O, Pugliese M, Boccuzzi G, Berta L \& Frairia R 2007 High energy shock waves activate 5 '-aminolevulinic Acid and increase permeability to Paclitaxel: antitumor effects of a new combined treatment on anaplastic thyroid cancer cells. Thyroid 17 91-99. (doi:10.1089/thy.2006.0142)

Cavalli R, Bisazza A, Trotta M, Argenziano M, Civra A, Donalisio M \& Lembo D 2012 New chitosan nanobubbles for ultrasound-mediated gene delivery: preparation and in vitro characterization. International Journal of Nanomedicine 7 3309-3318. (doi:10.2147/IJN.S30912)

Cavalli R, Soster M \& Argenziano M 2016 Nanobubbles: a promising efficient tool for therapeutic delivery. Therapeutic Delivery 7 117-138. (doi:10.4155/tde.15.92)

Chatterjee K, Zhang J, Honbo N \& Karliner JS 2010 Doxorubicin cardiomyopathy. Cardiology 115 155-162. (doi:10.1159/000265166)

Collis J, Manasseh R, Liovic P, Tho P, Ooi A, Petkovic-Duran K \& Zhu Y 2010 Cavitation microstreaming and stress fields created by microbubbles. Ultrasonics 50 273-279. (doi:10.1016/j. ultras.2009.10.002)

Diederich CJ \& Hynynen K 1999 Ultrasound technology for hyperthermia. Ultrasound in Medicine and Biology 25 871-887. (doi:10.1016/S0301-5629(99)00048-4)

Du L, Jin Y, Zhou W \& Zhao J 2011 Ultrasound-triggered drug release and enhanced anticancer effect of doxorubicin-loaded poly(D,Llactide-co-glycolide)-methoxy-poly(ethylene glycol) nanodroplets.
Ultrasound in Medicine and Biology 37 1252-1258. (doi:10.1016/j. ultrasmedbio.2011.05.012)

Fan X, Wang L, Guo Y, Xiong X, Zhu L and Fang K 2016 Inhibition of prostate cancer growth using doxorubicin assisted by ultrasoundtargeted nanobubble destruction. International Journal of Nanomedicine 11 3585-3596. (doi:10.2147/IJN.S111808)

Fang J, Nakamura H \& Maeda H 2011 The EPR effect: Unique features of tumor blood vessels for drug delivery, factors involved, and limitations and augmentation of the effect. Advanced Drug Delivery Reviews 63 136-151. (doi:10.1016/j.addr.2010.04.009)

Frairia R, Catalano MG, Fortunati N, Fazzari A, Raineri M \& Berta L 2003 High energy shock waves (HESW) enhance paclitaxel cytotoxicity in MCF-7 cells. Breast Cancer Research and Treatment $\mathbf{8 1}$ 11-19. (doi:10.1023/A:1025477421467)

Gao Z, Kennedy AM, Christensen DA \& Rapoport NY 2008 Drug-Loaded Nano/microbubbles for combining ultrasonography and targeted chemotherapy. Ultrasonics 48 260-270. (doi:10.1016/j. ultras.2007.11.002)

Giuffrida D \& Gharib H 2000 Anaplastic thyroid carcinoma: current diagnosis and treatment. Annals of Oncology 11 1083-1089. (doi:10.1 023/A:1008322002520)

Golla K, Bhaskar C, Ahmed F \& Kondapi AK 2013 A target-specific oral formulation of Doxorubicin-protein nanoparticles: efficacy and safety in hepatocellular cancer. Journal of Cancer 4 644-652. (doi:10.7150/jca.7093)

Guo DD, Xu CX, Quan JS, Song CK, Jin H, Kim DD, Choi YJ, Cho MH \& Cho CS 2009 Synergistic anti-tumor activity of paclitaxelincorporated conjugated linoleic acid-coupled poloxamer thermosensitive hydrogel in vitro and in vivo. Biomaterials $\mathbf{3 0}$ 4777-4785. (doi:10.1016/j.biomaterials.2009.05.051)

Haddad RI, Lydiatt WM, Ball DW, Busaidy NL, Byrd D, Callender G, Dickson P, Duh QY, Ehya H, Haymart M et al. 2015 Anaplastic Thyroid Carcinoma, Version 2.2015. Journal of the National Comprehensive Cancer Network 13 1140-1150. (doi:10.6004/ jnccn.2015.0139)

Hinz B 2007 Formation and function of the myofibroblast during tissue repair. Journal of Investigative Dermatology 127 526-537. (doi:10.1038/ sj.jid.5700613)

Kodama T, Doukas AG \& Hamblin MR 2002 Shock wave-mediated molecular delivery into cells. Biochimica et Biophysica Acta 1542 186-194. (doi:10.1016/S0167-4889(01)00177-X)

Krstić J, Trivanović D, Mojsilović S \& Santibanez JF 2015 Transforming growth factor-beta and oxidative stress interplay: implications in tumorigenesis and cancer progression. Oxidative Medicine and Cellular Longevity 2015 654594. (doi:10.1155/2015/654594)

Kuwahara F, Kai H, Tokuda K, Kai M, Takeshita A, Egashira K \& Imaizumi T 2002 Transforming growth factor-beta function blocking prevents myocardial fibrosis and diastolic dysfunction in pressure overloaded rats. Circulation 106 130-135. (doi:10.1161/01. CIR.0000020689.12472.E0)

Lauer U, Bürgelt E, Squire Z, Messmer K, Hofschneider PH, Gregor M \& Delius M 1997 Shock wave permeabilization as a new gene transfer method. Gene Therapy 4 710-715. (doi:10.1038/sj.gt.3300462)

Lin W, Xie X, Deng J, Liu H, Chen Y, Fu X, Liu H \& Yang Y 2016 Cellpenetrating peptide-doxorubicin conjugate loaded NGR-modified nanobubbles for ultrasound triggered drug delivery. Journal of Drug Targeting 24 134-146. (doi:10.3109/1061186X.2015.1058802)

Marano F, Argenziano M, Frairia R, Adamini A, Bosco O, Rinella L, Fortunati N, Cavalli R \& Catalano MG 2016 Doxorubicin-loaded nanobubbles combined with extracorporeal shock waves: basis for a new drug delivery tool in anaplastic thyroid cancer. Thyroid $\mathbf{2 6}$ 705-716. (doi:10.1089/thy.2015.0342)

Minotti G, Menna P, Salvatorelli E, Cairo G \& Gianni L 2004 Anthracyclines: molecular advances and pharmacologic developments in antitumor activity and cardiotoxicity. Pharmacological Reviews 56 185-229. (doi:10.1124/pr.56.2.6) http://erc.endocrinology-journals.org DOI: 10.1530/ERC-17-0045
๑) 2017 Society for Endocrinology Printed in Great Britain 
Mukhopadhyay P, Rajesh M, Bátkai S, Kashiwaya Y, Haskó G, Liaudet L, Szabó C \& Pacher P 2009 Role of superoxide, nitric oxide, and peroxynitrite in doxorubicin-induced cell death in vivo and in vitro. American Journal of Physiology: Heart and Circulatory Physiology 296 H1466-H1483. (doi:10.1152/ajpheart.00795.2008)

Palmero A, Berger M, Venturi C, Ferrero I, Rustichelli D, Berta L, Frairia R, Madon E \& Fagioli F 2006 High energy shock waves enhance the cytotoxic effect of doxorubicin and methotrexate to human osteosarcoma cell lines. Oncology Reports 15 267-273. (doi:10.3892/ or.15.1.267)

Rassweiler JJ, Knoll T, Köhrmann KU, McAteer JA, Lingeman JE, Cleveland RO, Bailey MR \& Chaussy C 2011 Shock wave technology and application: an update. European Urology 59 784-796. (doi:10.1016/j.eururo.2011.02.033)

Razavi-Azarkhiavi K, Jafarian AH, Abnous K, Razavi BM, Shirani K, Zeinali M, Jaafari MR \& Karimi G 2016 The comparison of biodistribution, efficacy and toxicity of two PEGylated liposomal doxorubicin formulations in mice bearing C-26 colon carcinoma: a preclinical study. Drug Research 66 330-336. (doi:10.1055/s-0035-1569447)

Schweppe RE, Klopper JP, Korch C, Pugazhenthi U, Benezra M, Knauf JA, Fagin JA, Marlow LA, Copland JA, Smallridge RC et al. 2008 Deoxyribonucleic acid profiling analysis of 40 human thyroid cancer cell lines reveals cross-contamination resulting in cell line redundancy and misidentification. Journal of Clinical Endocrinology and Metabolism 93 4331-4441. (doi:10.1210/jc.2008-1102)

Sheppard RJ, Berger J \& Sebag IA 2013 Cardiotoxicity of cancer therapeutics: current issues in screening, prevention, and therapy. Frontiers in Pharmacology 4 19. (doi:10.3389/fphar.2013.00019)

Singal PK, Li T, Kumar D, Danelisen I \& Iliskovic N 2000 Adriamycininduced heart failure: mechanism and modulation. Molecular and Cellular Biochemistry 20 77-86. (doi:10.1023/A:1007094214460)

Sinha BK, Katki AG, Batist G, Cowan KH \& Myers CE 1987 Adriamycinstimulated hydroxyl radical formation in human breast tumor cells. Biochemical Pharmacology 36 793-796. (doi:10.1016/00062952(87)90164-X)
Smallridge RC \& Copland JA 2010 Anaplastic thyroid carcinoma: pathogenesis and emerging therapies. Journal of Clinical Oncology 22 486-497. (doi:10.1016/j.clon.2010.03.013)

Smallridge RC, Ain KB, Asa SL, Bible KC, Brierley JD, Burman KD, Kebebew E, Lee NY, Nikiforov YE, Rosenthal MS et al. 2012 American Thyroid Association guidelines for management of patients with anaplastic thyroid cancer. Thyroid 22 1104-1039. (doi:10.1089/thy.2012.0302)

Spallarossa P, Altieri P, Garibaldi S, Ghigliotti G, Barisione C, Manca V, Fabbi P, Ballestrero A, Brunelli C, Barsotti A 2006 Matrix metalloproteinase- 2 and -9 are induced differently by doxorubicin in H9c2 cells: the role of MAP kinases and NAD(P)H oxidase. Cardiovascular Research 69 736-745. (doi:10.1016/j. cardiores.2005.08.009)

Tacar O, Sriamornsak P \& Dass CR 2013 Doxorubicin: an update on anticancer molecular action, toxicity and novel drug delivery systems. Journal of Pharmacy and Pharmacology 65 157-170. (doi:10.1111/j.2042-7158.2012.01567.x)

Wang CJ 2012 Extracorporeal shockwave therapy in musculoskeletal disorders. Journal of Orthopaedic Surgery and Research 711. (doi:10.1186/1749-799X-7-11)

Wohlfart S, Khalansky AS, Gelperina S, Maksimenko O, Bernreuther C, Glatzel M \& Kreuter J 2011 Efficient chemotherapy of rat glioblastoma using doxorubicin-loaded PLGA nanoparticles with different stabilizers. PLoS One 6 e19121. (doi:10.1371/journal.pone.0019121)

Yu Y, Chen CK, Law WC, Weinheimer E, Sengupta S, Prasad PN \& Cheng C 2014 Polylactide-graft-doxorubicin nanoparticles with precisely controlled drug loading for $\mathrm{pH}$-triggered drug delivery. Biomacromolecule 15 524-532. (doi:10.1021/bm401471p)

Zhang S, Liu X, Bawa-Khalfe T, Lu LS, Lyu YL, Liu LF \& Yeh ET 2012 Identification of the molecular basis of doxorubicin-induced cardiotoxicity. Nature Medicine 18 1639-1642. (doi:10.1038/nm.2919)

Zhao Y, McLaughlin D, Robinson E, Harvey AP, Hookham MB, Shah AM, McDermott BJ \& Grieve DJ 2010 Nox2 NADPH oxidase promotes pathologic cardiac remodeling associated with doxorubicin chemotherapy. Cancer Research 70 9287-9297. (doi:10.1158/00085472.CAN-10-2664)

Received in final form 14 February 2017

Accepted 4 April 2017

Accepted Preprint published online 7 April 2017
(C) 2017 Society for Endocrinology Printed in Great Britain
Published by Bioscientifica Ltd 